\title{
HVDLP : HORIZONTAL VERTICAL DIAGONAL LOCAL PATTERN BASED FACE RECOGNITION
}

\author{
ChandrakalaV ${ }^{1}$, Venu $\mathrm{Kumar}^{2}$, K Suresh Babu ${ }^{2}, \mathrm{~K}$ B Raja ${ }^{2}$, Venugopal K R ${ }^{3}$ \\ ${ }^{1}$ Dr. Ambedkar Institute of Technology, Bangalore -560056, India. \\ ${ }^{2}$ University of Visvesvaraya College of Engineering, \\ Bangalore University, Bangalore-560 001, India
}

\begin{abstract}
Face image is an efficient biometric trait to recognize human beings without expecting any co-operation from a person. In this paper, we propose HVDLP: Horizontal Vertical Diagonal Local Pattern based face recognition using Discrete Wavelet Transform (DWT) and Local Binary Pattern (LBP). The face images of different sizes are converted into uniform size of 108×990and color images are converted to gray scale images in pre-processing. The Discrete Wavelet Transform (DWT) is applied on pre-processed images and $L L$ band is obtained with the size of $54 \times 45$. The Novel concept of HVDLP is introduced in the proposed method to enhance the performance. The HVDLP is applied on $9 \times 9$ sub matrix of LL band to consider HVDLP coefficients. The local Binary Pattern $(L B P)$ is applied on HVDLP of LL band. The final features are generated by using Guided filters on HVDLP and LBP matrices. The Euclidean Distance (ED) is used to compare final features of face database and test images to compute the performance parameters.
\end{abstract}

\section{KEYWORDS}

Biometric; DWT, LBP, HVDLP, Face Recognition

\section{INTRODUCTION}

An automatic face images are identified or verified by comparing features of test image and database images using classifiers. The face recognition model has three modules viz., (i)Enrolment Module (ii)Test Module and (iii)Matching Module. The enrolment module has two sections such as pre-processing section and feature extraction section. In pre-processing section, the colour images are converted into grey scale images, images are resized and qualities of images are enhanced using appropriate filters or histogram equalization techniques. In feature extraction section, there are three main methods used to extract features are spatial domain, transform domain and hybrid domain techniques. In spatial domain techniques, the image pixels are directly considered and manipulated without converting into other domains. Few examples are (i) Mean, Median, Standard Deviation and Variance of pixel intensity values. (ii) Principle Component Analysis (iii) Linear Binary Pattern (LBP) and Singular Value Decomposition etc., In Transform domain techniques, the image pixel intensity values are converted into other domains and the computed co-efficients are manipulated. Few examples are (i) Fast Fourier Transform (ii) Discrete Cosine Transform (iii) Short Time Fourier Transform (iv) Discrete Wavelet Transform 
Signal \& Image Processing : An International Journal (SIPIJ) Vol.8, No.5, October 2017

etc., In hybrid domain the spatial and transform domain techniques are combined to extract features.

The test module has pre-processing and feature extraction sections, which are similar to enrolment module. In matching section, the features of database images are compared with test image features using the distance formulae or classifiers. The distance formulae used are Euclidean distance (ED), Hamming Distance, Chi-square distance etc., The classifiers used are Neural Networks, Support Vector Machines, Self Organized mapping, Random Forest etc.,

Contribution: In this paper, face recognition based on horizontal, vertical and diagonal local pattern is proposed. The local binary pattern is applied HVDLP of LL band. The final features are from guided filters applied on HVDLP and LBP matrices to obtain better recognition rate.

Organization: This paper is organized as follows: Section 2 describes the overview of literature survey, Section 3 focuses on proposed model, Section 4 describes proposed algorithm, and Section 5 displays the Performance analysis and results using HVDLP, Finally Section 6 presents the conclusion and outlines of future work.

\section{LITERATURE SURVEY}

In this section, the existing various approches of biometrics are discussed. Shengcai Liao et al., [1] proposed Learning Multi-Scale Block Local Binary Patterns for Face Recognition. In this model Multiscale Block Local Binary Pattern (MB-LBP) is performed on average values of block sub region instead of individual pixel and uniform patterns are defined via statistical analysis. To construct face classifiers and to select MB-LBP features Ada boost learning method is used. Experiments are conducted on Face Recognition Grand Challenge (FRGC) ver2.0. Abdallah et al., [2] proposed Avatar Face Recognition using Wavelet Transform and Hierarchical Multi-scale LBP (HMLBP). The technique consists of three stages: i) preprocessing ii) Feature extraction iii) Recognition. i) The wavelet decomposition enhances the common features in the same class of image. ii) HMLBP extracts the representative features without training from each avatar face image. iii) Chi- Square distance makes the decision and indicates the correct class to which the input image belongs to. Timo Ojala and Matti Pietika Ėinen[3] proposed Multiresolution GrayScale and Rotation Invariant Texture Classification with Local Binary Patterns. This method recognizes local binary patterns and these are the basic properties of local image texture. The occurrence histogram is very powerful texture features, these are derived by using Multiresolution operators which are genelarized from gray-scale and rotation invariant, they utilize the method which combines multiple operators that detects the patterns for the quantization of the angular space, using invariant operator and for any spatial resolution invariant operators. Yu Feng et al., [4] presented kernel embedding Multi Orientation Local Pattern [MOLP] on local binary pattern and image gradient orientations, next the histogram feature takes the sign component and magnitude component on each orientation. The refined features are fused in the kernel embedding discriminates subspace learning model. The experiments are applied on different traits such as face, palm, object categorization etc., and shows that MLOP achieved better results. Navaneeth Bodla et al., [5] proposed non-linear high-dimensional projection of the deep features and generates the template feature information by using feature fusion heterogeneous technique on different face features. The experimental results show better and effective. Abdul Matin et al., [6] deals with the multimodal i.e., iris and face by applying Daugman and PCA for extraction of features and combining the normalized scores by weighted 
Signal \& Image Processing : An International Journal (SIPIJ) Vol.8, No.5, October 2017

score level fusion technique based on decision of accept or reject on the fused score of iris and face. The recognition technique of fusion is accurate and efficient. Aditya Gupta et al., [7] proposed a feature level fusion of face, palm vein and palmprint modalities using DCT for feature extraction to calculate the standard deviation and stored as feature vector. Results showed better result in multimodal rather in unimodal. Zhao et al., [8] presented a survey on machine recognition of human faces having still images and video images, Methodologies deployed in all the categories, characteristics and their pros and cons.

In addition provided summaries of current developments and challenging issues.Ying et al., [9] introduced to handle different pose variations in 2D face images and develop a regression model that does face alignment, pose correction and face representation by the Orthogonal Procrustes Problem (OPP). By adopting a progressive strategy and in each regression the orthogonal matrix computes the linear transformation between near-by viewpoints. The pose variations are dealt only in horizontal direction. The experimental results were better by using misaligned test images with pose variations regardless of the training images are frontal or non-frontal. Xiujuan Chai et al., [10] proposed Locally Linear Regression (LLR) method generates frontal view from the nonfrontal face image with the assumption that there exists an approximate linear mapping between a non frontal face image and frontal. The proposed algorithm initially performs the dense sampling in the non frontal face image to obtain overlapped patches. The Linear regression is applied on each patch for the prediction of its virtual frontal patch. Then through the combination of all the patches, virtual frontal view is generated. Ralph Gross et al., proposed in appearance based object recognition features are the pixel intensity values and it is corresponding directly to the light emitted from the object called plenoptic function or light field, applicable to all the images. All of the images which ever they come from estimate the light field of the object. This field is used in appearance based face recognition. Smriti Tikoo and Nitin Malik [12] presented facial detection using viola Jones algorithm. It consists of i) Haar feature selection ii) Creating an integral image iii) Adaboost training iv) Cascading amplifiers and recognition of face has been done by using Back Propagation Neural Network (BPNN). Anagha S Dhavalikar and Kulkarni [13] proposed Automatic Facial Expression Recognition system comprises of three stages i) Face detection involves skin color detection using $\mathrm{YCbCr}$ color model, lighting compensation and morphological operations ii) Feature extraction extracts $n$ facial features of eyes, nose and mouth using Active Appearance Model iii) Facial recognition using Euclidean Distance. The recognition rate was better. Kavitha and Manjeet Kaur [14] analyzed different algorithms and methods. Samah YAHIA et al., [15] proposed 3D Local Binary Pattern 3D Grey Level Cooccurrence Matrix and combination of both. Initially training phase is required to pass the test phase and Multiclass Support Vector Machine for classification. The performance result was better.

\section{PROPOSED MODEL}

The Face recognition model is proposed based on DWT, HVDP, and LBP is as shown in the figure 1 . The novel concept of diagonal, vertical and horizontal pattern co-efficients of $9 \times 9$ cell is introduced to extract features.

\subsection{Face Databases}

The various databases such as ORL, JAFFE, Indian Female, Indian Male and L-Speck are used to test the model for performance analysis. 
Signal \& Image Processing : An International Journal (SIPIJ) Vol.8, No.5, October 2017

3.1.1. Olivetti Research Laboratory (ORL) [16]: The database has forty persons with ten images per person i.e., it has four hundred images in total. The face images are captured with different lighting conditions, facial expressions, timings and angles with each image of size $92 \times 112$. The face image samples of a person is as shown in the figure 2 .

3.1.2. Indian Female [17]: The database has eleven persons with twenty-two images per person i.e., it has two hundred forty images in total. The images are captured based on facial orientations with different emotional expressions with each image of size $640 \times 480$. The face image samples of a person is as shown in the figure 3 .

3.1.3. Indian Male [17]: The face database of two hundred and twenty images of eleven persons. The face images are captured on different orientation angles with various expressions with each image of size $640 \times 480$. The face image samples of a person are as shown in the figure 4 .

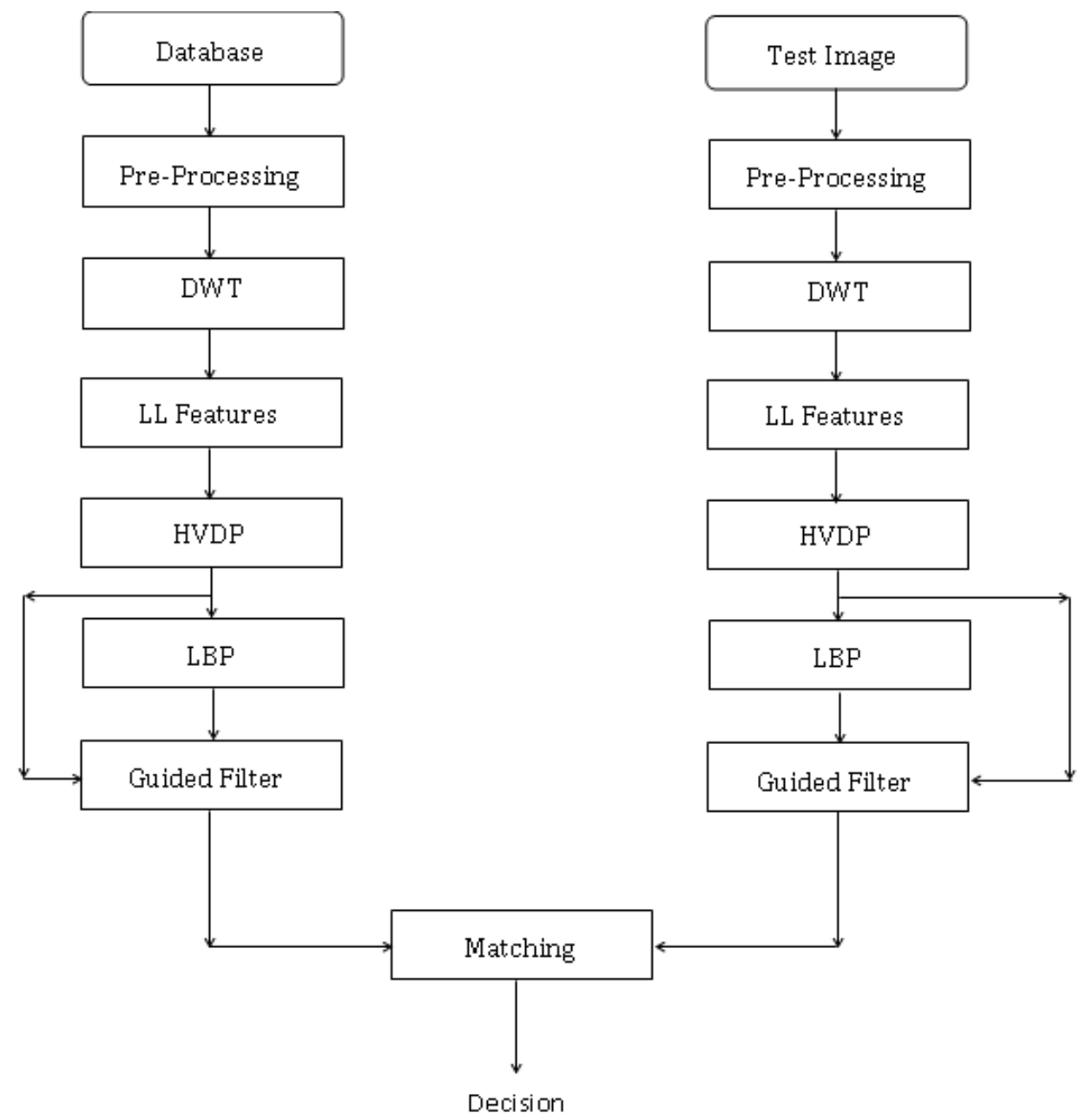

Fig. 1: Proposed Model 
Signal \& Image Processing : An International Journal (SIPIJ) Vol.8, No.5, October 2017
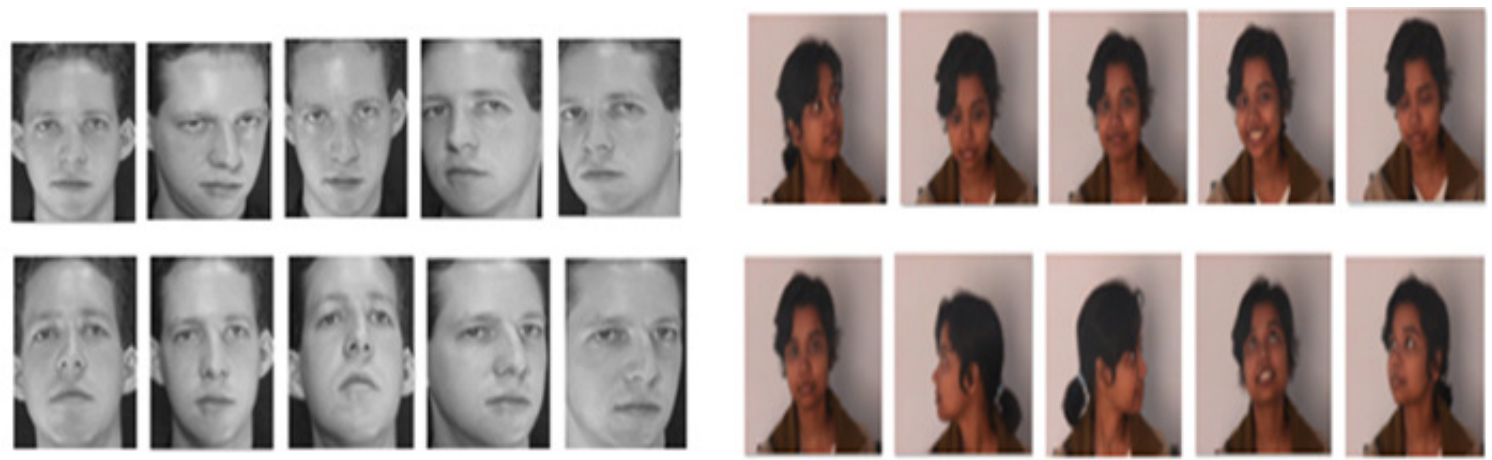

Fig 2: ORL face database

Fig 3: Indian female database
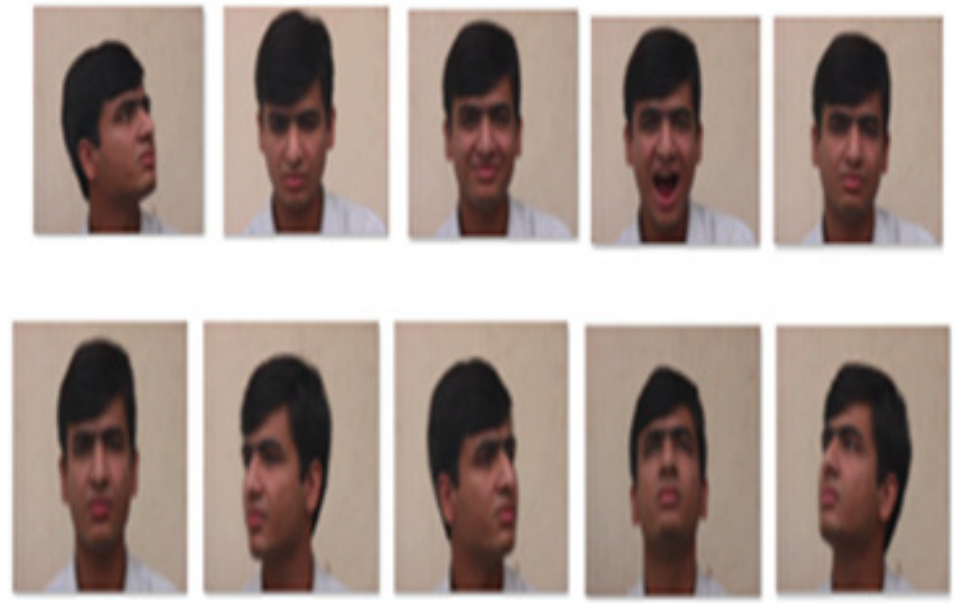

Fig 4: Indian male database

3.2 Pre-Processing: The face image databases have different dimensions; hence the dimensions are altered according to the requirements. The size of Indian male and Indian females databases are high, hence it is reduced. The size of each face image is resized to $108 \times 90$ for all face databases, which is suitable to generate LL co-efficient for HVDP method

3.3 Discrete Wavelet Transform (DWT): [18] The Discrete Wavelet Transformation is used to obtain i) Frequency resolution ii) Spatial resolution for low and high frequencies respectively as shown in figure 5. The image is classified into approximation and detailed sub bands. The approximation sub band has significant information of an image. The latter sub band has information on horizontal, vertical and diagonal details. The low pass filters and high pass filters are generated. The four sub bands such as LL, LH, HL and HH band formed by Low pass filter and Low pass filters, Low pass and High pass filters, High pass and Low pass filters, and High pass and High pass filters.

The DWT is applied on face image to obtain four sub bands as and the corresponding image sub bands as shown in figure 6. The LL sub band shows significant information of an original face image. The detailed sub bands of DWT give vertical, horizontal and diagonal edge information's of original image. Each sub band dimensions are $54 \times 45$. 
Signal \& Image Processing : An International Journal (SIPIJ) Vol.8, No.5, October 2017

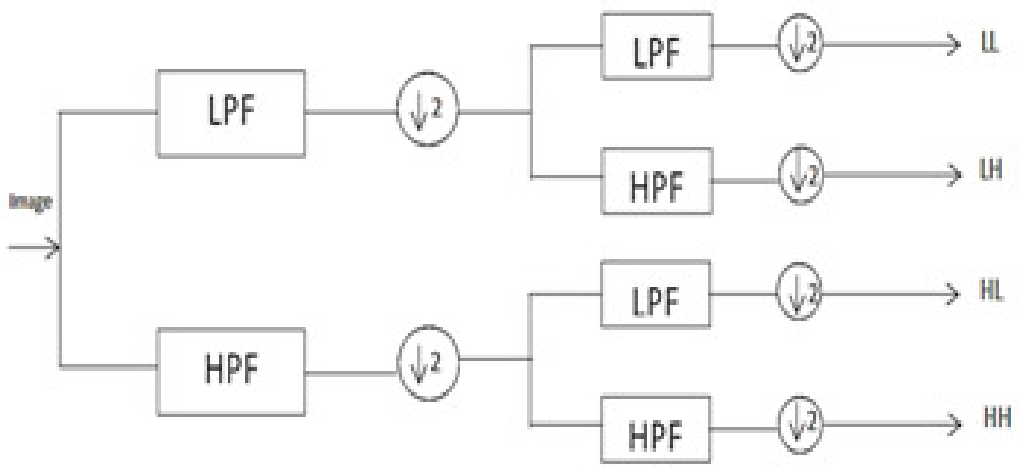

Fig 5: One level 2D-DWT

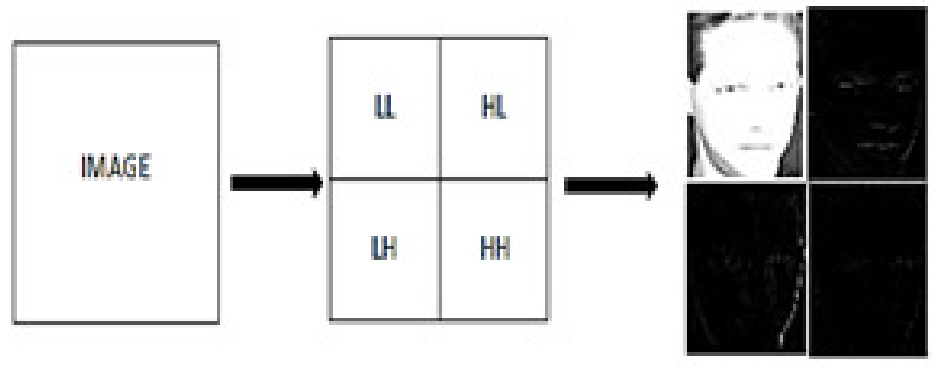

a) Original image b) Decomposition c) Image composition

Fig 6: DWT Decomposition

3.4 Horizontal Vertical Diagonal Local Pattern (HVDLP): The new local binary pattern is introduced by considering $9 \times 9$ image matrix cell, which leads to HVDLP.

The four vectors such as centre vertical vector, centre horizontal vector, left diagonal vector and right diagonal vectors are considered as pattern vectors in each cell to extract features as shown in figure 7. Each vector has nine co-efficients. The four vectors of each cell are as follows.

$\mathrm{LD}=$ Left diagonal vector $=\left[\mathrm{ld}_{1}, \mathrm{ld}_{2}, \mathrm{ld}_{3}\right.$ $\left.\operatorname{ld}_{9}\right]$

$\mathrm{CV}=$ Centre vertical vector $=\left[\mathrm{cv}_{1}, \mathrm{cv}_{2}, \mathrm{cv}_{3}\right.$ $\left.\mathrm{cv}_{9}\right]$

$\mathrm{RD}=$ Right diagonal vector $=\left[\mathrm{rd}_{1}, \mathrm{rd}_{2}, \mathrm{rd}_{3}\right.$ $\left.\mathrm{rd}_{9}\right]$

$\mathrm{CH}=$ Centre horizontal vector $=\left[\mathrm{ch}_{1}, \mathrm{ch}_{2}, \mathrm{ch}_{3}\right.$ $\left.\mathrm{ch}_{9}\right]$

Each $9 \times 9$ cell has 36 co-efficient and the co-efficient of first cell are given in expression 5 . 
Signal \& Image Processing : An International Journal (SIPIJ) Vol.8, No.5, October 2017

cell $=\left[\begin{array}{l}L D 1 \\ C V 1 \\ R D 1 \\ C H 1\end{array}\right]=\left[\begin{array}{llllll}L d 1 & L d 2 & L d 3 & \ldots \ldots . . & L d 9 \\ C v 1 & C v 2 & C v 3 & \ldots . . . . & C v 9 \\ r d 1 & r d 2 & r d 3 & \ldots . . . & & r d 9 \\ C h 1 & C h 2 & C h 3 & . . . . . . & C h 9\end{array}\right]$

$4 \times 9$

The $4 \times 9$ matrix of first cell is converted into column vector, which represent HVDLP features of cell1 as shown in expression 6

$H V D P$ of cell $1=\left[\begin{array}{c}1 L d 1 \\ \cdot \\ \cdot \\ 1 L d 9 \\ 1 C v 1 \\ \cdot \\ \cdot \dot{C v 9} \\ 1 R d 1 \\ \cdot \\ \cdot \\ 1 R d 9 \\ 1 \operatorname{ch} 1 \\ \cdot \\ \cdot \\ 1 C h 9\end{array}\right]$

$(36 \times 1)$

Where

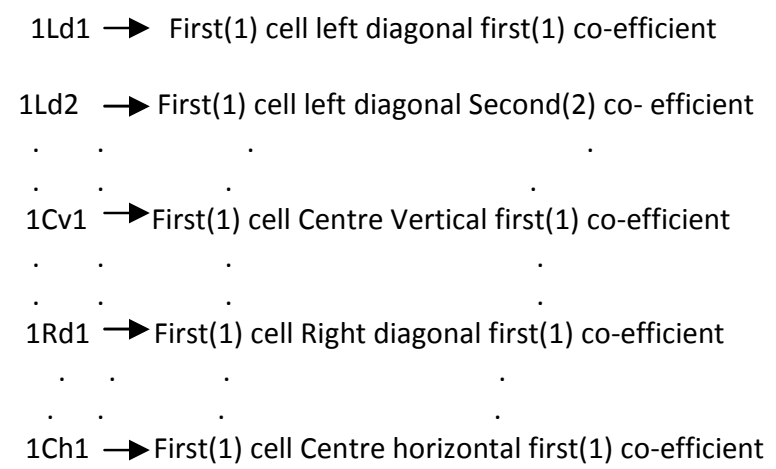

The total number of co-efficient usage for full matrix is only $44.4 \%$ with HVDLP, hence this technique require less memory and also increases computation speed. 
Signal \& Image Processing : An International Journal (SIPIJ) Vol.8, No.5, October 2017

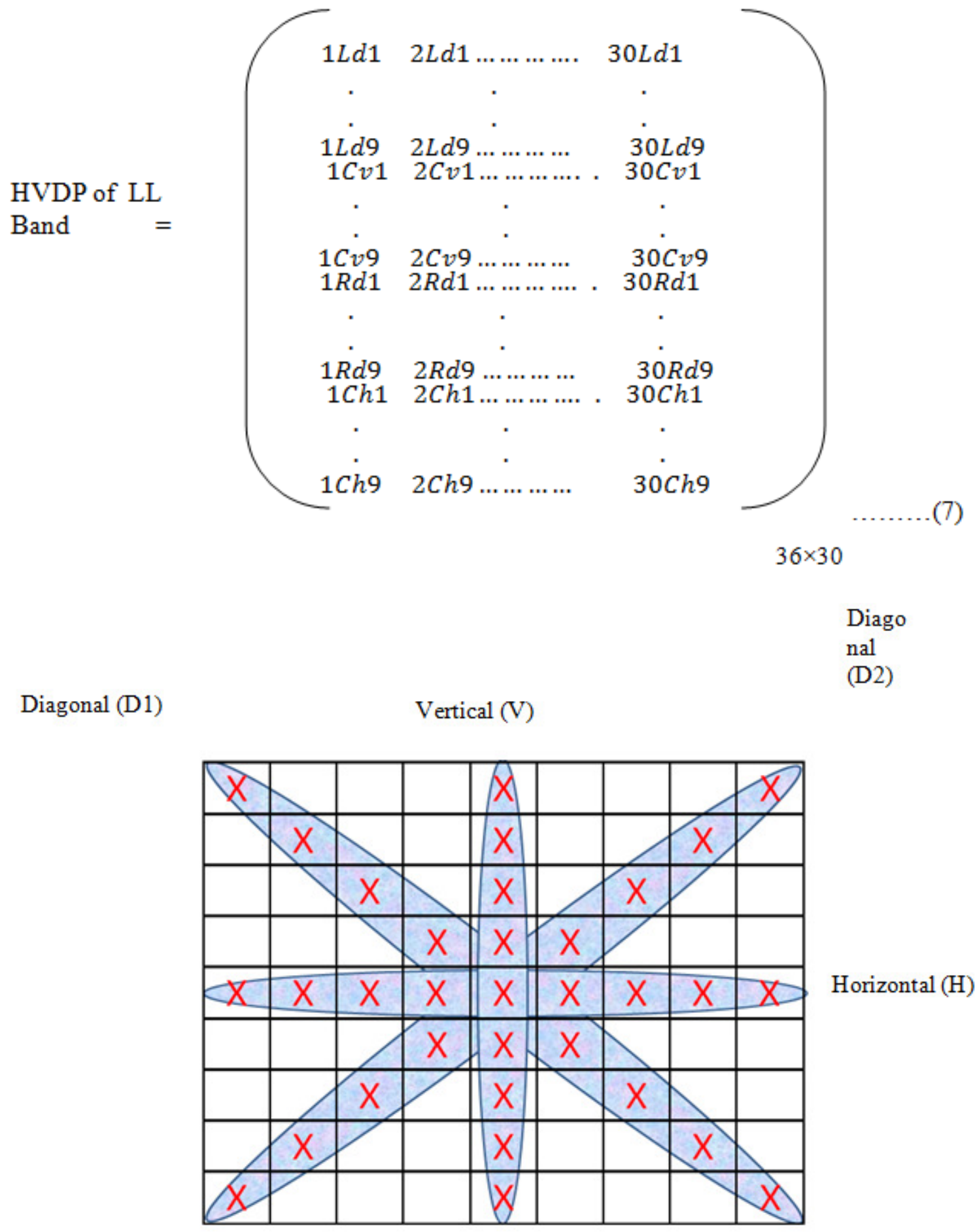

Fig. 7 HVDLP Pixel 
Signal \& Image Processing : An International Journal (SIPIJ) Vol.8, No.5, October 2017

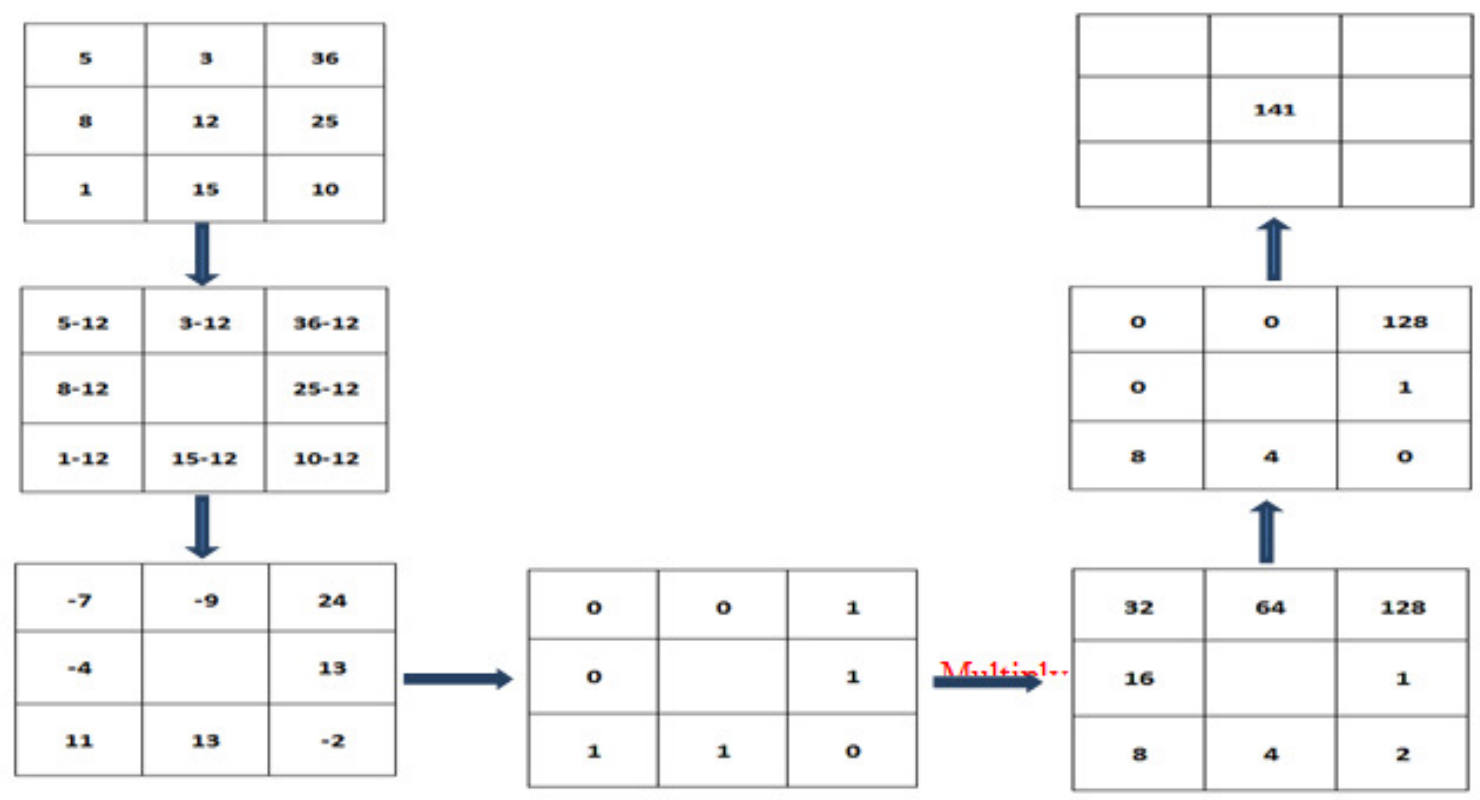

Fig. 8. Decimal value labeling

3.5 Local Binary Pattern (LBP): [19] Texture analysis is important in biometric image analysis and classifications based on local spatial domain intensity variations.

The LBP is invariant to illumination variations. The $3 \times 3$ matrix is an image is considered and the intensity value pixel values. The Neighbourhood Pixel (NP) values are compared with Centre Pixel(CP) values and converted into Binary Values(BV) based on the equation 1.

$B V=\left\{\begin{array}{lc}1 & N P>C P \\ 0 & N P<C P\end{array}\right.$

Similarly, all eight neighbourhood pixel intensity values are converted into binary values and a set of eight binary values are converted into decimal values, which is assigned to centre pixel. The procedure is continued for all $3 \times 3$ matrix and replace all pixel values by LBP equivalent values to obtain LBP image of original face image.

The illustration of converting centre pixel into LBP equivalent value is shown in figure 8 .

3.6 Guided Filtering: [20] It is derived from Local linear model. The information transfer from one image to another image takes place in significant way.

\section{ALGORITHM}

Problem Definition: The face recognition based on DWT, HVDP, LBP and guided filter is proposed to identify human beings effectively. The performance parameters such as percentage of FRR, FAR, Max. TSR, Opt. TSR and EER are evaluated and compared with existing algorithms to validate the efficiency of the proposed method. 
Signal \& Image Processing : An International Journal (SIPIJ) Vol.8, No.5, October 2017

Objectives: The face recognition model is proposed with the following objectives

(i) To obtain maximum Opt. TSR and Max. TSR

(ii) To obtain minimum values of EER

The proposed algorithm is as shown in table 1

Table 1: proposed algorithm

Input: Face images from various databases
Output: recognition of face images
1. Face images are resized to $108 \times 90$
2. The DWT is applied on resized image to obtain LL band
coefficients and dimension of LL band is $54 \times 45$
3.The HVDP concept is used on LL band and dimension is $36 \times 30$
4.The LBP is applied on HVDP matrix
5.The guided filter is used on HVDP and LBP to get better features.

\section{Performance Analysis}

In this section, the definitions of performance parameters, the performance evaluation and comparison of proposed method with existing methods are discussed.

5.1 Definitions of Performance Parameters: The evaluation of performance parameters such as FRR, FAR, TSR and EER is given.

5.1.1 False Rejection Ration (FRR): The ratio of number of genuine persons rejected to the total number of PID as given in equation 3.

$\mathrm{FRR}=\frac{\text { Number of genuine persons in the database rejected }}{\text { Total number of persons inside the database }}$

5.1.2 False Acceptance Ratio (FAR): The ratio of number of imposter persons accepted as genuine to the total number of persons outside the database as given in equation 4 .

$\mathrm{FAR}=\frac{\text { Number of imposter persons accepted as genuine }}{\text { Total number of persons outside the database }}$

5.1.3 Total Success Rate (TSR): The ratio of number of genuine persons recognized correctly to the total number of persons inside the database as given in equation 5 . 
Signal \& Image Processing : An International Journal (SIPIJ) Vol.8, No.5, October 2017

$\mathrm{TSR}=\frac{\text { Number of the genuine persons recognized correctly }}{\text { Total number of the persons inside the database }}$

5.1.4 Equal Error Rate (EER): The optimum error between the FAR and FRR. The value of EER is intersection of FRR and FAR. The lower EER values gives the better efficiency of an algorithm.

5.2 Performance Evaluation using Various Face Databases: The database is created by considering different combination of Persons Inside the Database (PID) and Persons Outside the Database (POD) to observe the variation of FRR, FAR, TSR and EER for different threshold values of the proposed model. The face databases such as ORL, Indian Female and Indian Male are used to test the performance of the proposed method.

5.2.1 ORL Face Database: The variations of performance parameters such as percentage Optimum TSR (Opt. TSR), Maximum TSR (Max TSR) and EER for different combinations of PID and POD are tabulated in Table 2

Table 2. Variations of performance parameters with PID and POD for ORL database

\begin{tabular}{|c|c|c|c|c|}
\hline PID & POD & Opt TSR (\%) & Max TSR (\%) & EER (\%) \\
\hline $\mathbf{1 0}$ & 20 & 92.5 & 100 & 7.5 \\
\hline $\mathbf{1 2}$ & 28 & 90.4 & 100 & 9.6 \\
\hline $\mathbf{1 5}$ & 15 & 82 & 93.33 & 10.5 \\
\hline $\mathbf{2 0}$ & 20 & 78 & 90 & 16.5 \\
\hline
\end{tabular}

It is observed that as PID increases, the values of Opt. TSR and Max. TSR decreases and the percentage of EER values increases. The graphical representation for PID and POD combinations of 10 and 20 percentage variations is shown in figure 10. The value of FRR decreases with increase in threshold values. It is noticed that the percentage values of Opt. TSR, Max. TSR and EER values are $92.5,100$ and 7.5 respectively.

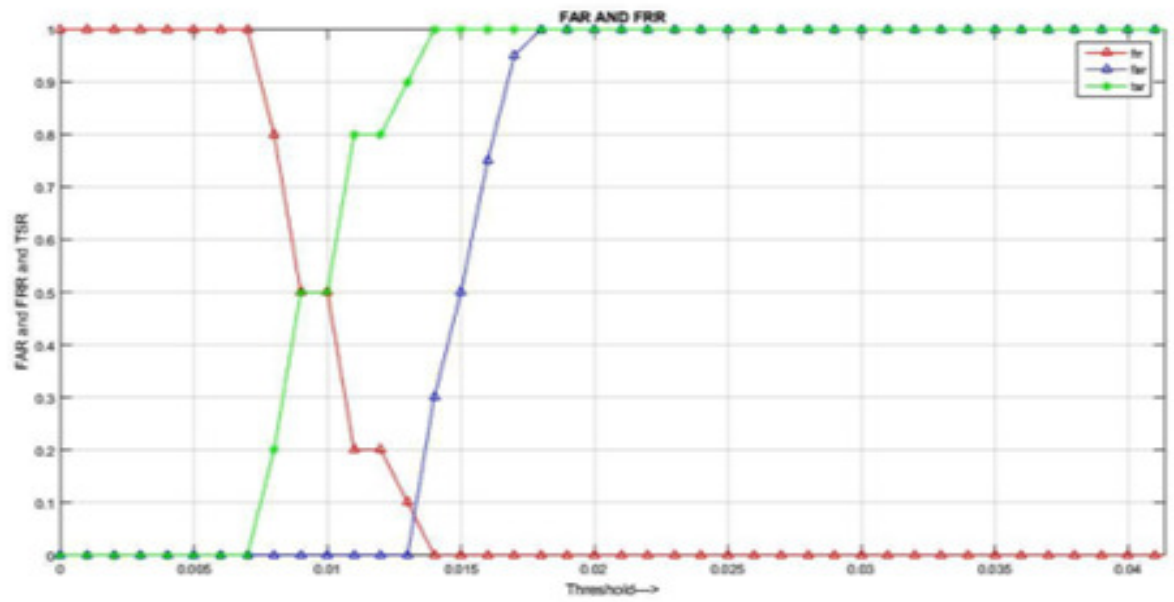

Fig 10: Variations of performance parameters for ORL with PID 10 POD 20 
Signal \& Image Processing : An International Journal (SIPIJ) Vol.8, No.5, October 2017

5.2.2 Indian Female: combinations of PID and POD are varied by considering Indian female face database to evaluate performance parameters. The variations of FRR, FAR and TSR with threshold are shown in figure 11 for PID 6. It is observed that the values of FRR decreases and the values of FAR and TSR increases with threshold values. The obtained values of Opt. TSR, Max. TSR and EER are 100,100 and 0 respectively. The different combinations of PID and POD are considered and noted the values of Opt. TSR, Max. TSR and EER are shown in table 3. The percentage of Opt. TSR values decreases and the percentage of EER values increases as PID values increases.

Table 3. Variations of performance parameters with PID and POD for Indian Female

\begin{tabular}{|c|c|c|c|c|}
\hline PID & POD & Opt TSR (\%) & Max TSR (\%) & EER (\%) \\
\hline 6 & 16 & 100 & 100 & 00 \\
\hline 11 & 11 & 91 & 100 & 09 \\
\hline 10 & 12 & 90 & 100 & 10 \\
\hline
\end{tabular}

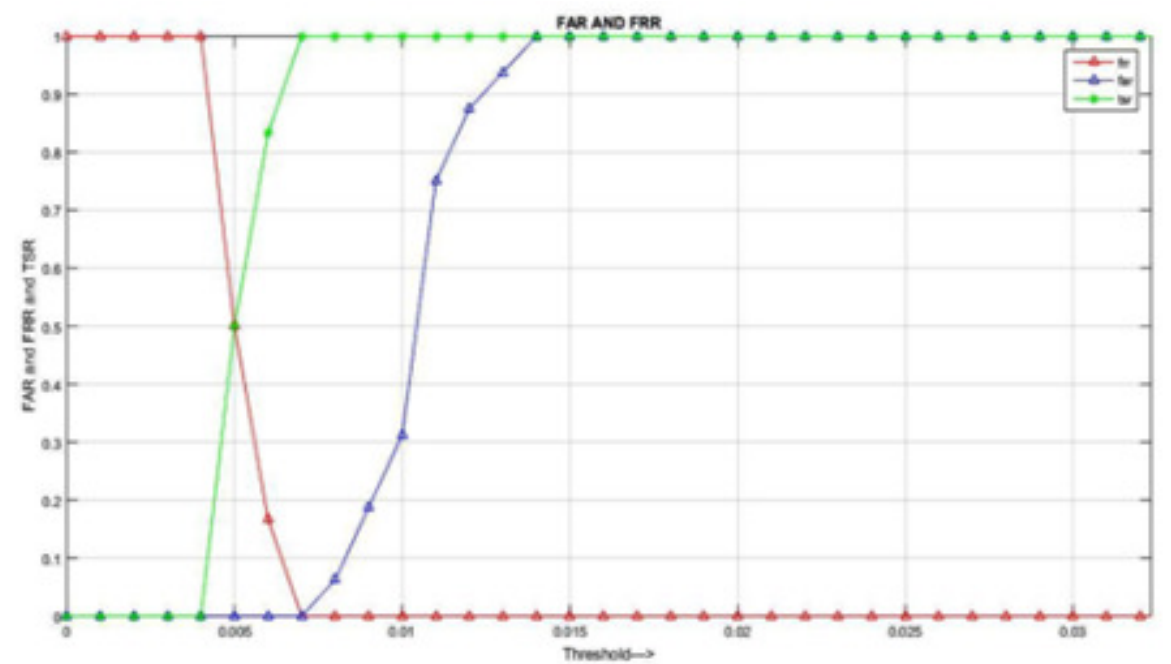

Fig 11: Variations of performance parameters for Indian Female PID 6POD 16 TSR 100\%

5.2.3 Indian Male: The combinations of PID and POD are varied by considering Indian male face database to evaluate performance parameters. The variations of FRR, FAR and TSR with threshold are shown in figure 12 for PID 6 and POD 14. It is observed that the values of FRR decreases and the values of FAR and TSR increases with threshold values. The obtained values of Opt. TSR, Max.TSR and EER are 61.1, 66.66 and 16.67 respectively. The different combinations of PID and POD are considered and noted the values of Opt. TSR, Max. TSR and EER are shown in table 4. The percentage of Opt. TSR values decreases and the percentage of EER values increases as PID values increases. 
Signal \& Image Processing : An International Journal (SIPIJ) Vol.8, No.5, October 2017

Table 4. Variations of performance parameters with PID and POD for Indian Male database

\begin{tabular}{|c|c|c|c|c|}
\hline PID & POD & Opt TSR (\%) & Max TSR (\%) & EER $(\%)$ \\
\hline 6 & 14 & 61.1 & 66.66 & 16.67 \\
\hline 8 & 12 & 61.25 & 62.5 & 16 \\
\hline 10 & 10 & 60 & 60 & 00 \\
\hline
\end{tabular}

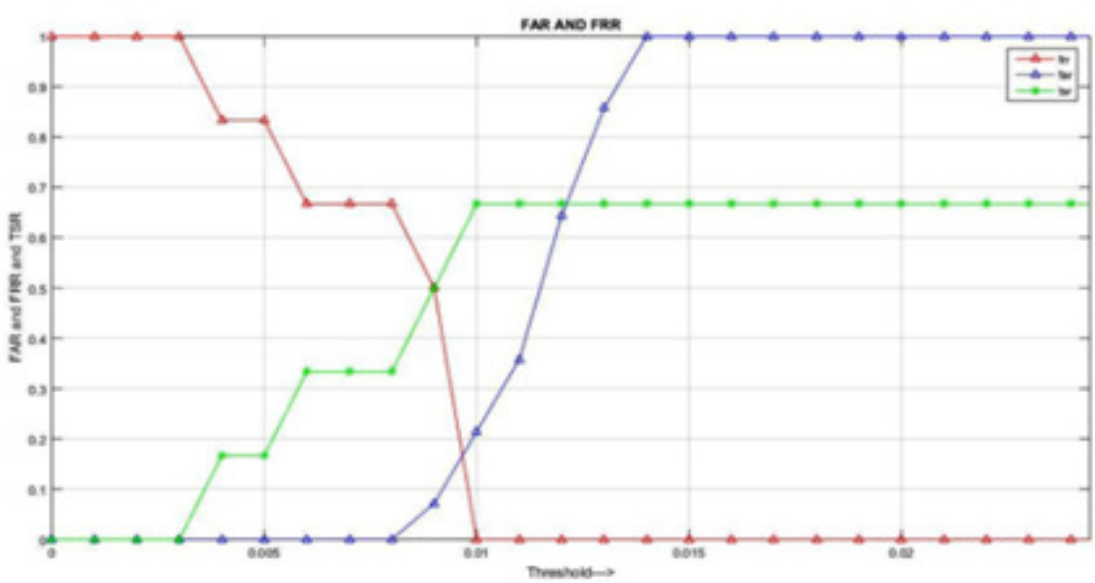

Fig 12: Variations of performance parameters for Indian Male with PID 6 POD 14

5.2.4 Comparison of proposed method with existing methods: The percentage of Opt. TSR and Max. TSR values of proposed method is compared with existing methods presented by Murugan et al., [21], Pallavi [22] and Sujatha [23]. It is observed that the performance of the proposed method is better compared to existing methodsas shown in table 5.

Table 5. Comparison of performance parameters

\begin{tabular}{|l|l|l|l|l|}
\hline SL No. & Authors & Techniques used & Opt TSR(\%) & Max TSR (\%) \\
\hline 1 & D Murugan et al., [21] & Gabor filter+DWT & 84.8 & 92 \\
\hline 2 & $\begin{array}{l}\text { Pallavi D.Wadkar et al., } \\
\text { [22] }\end{array}$ & DWT & 82.85 & 90 \\
\hline 3 & Sujatha B M et al., [23] & DWT+FFT+CLBP & 80 & 93.33 \\
\hline $\mathbf{4}$ & Proposed Model & $\begin{array}{l}\text { HVDBP+DWT+ } \\
\text { LBP }\end{array}$ & $\mathbf{9 2 . 5}$ & $\mathbf{1 0 0}$ \\
\hline
\end{tabular}

The proposed method is better compared to existing methods for the following reasons

1. The high frequency components of original face images are eliminated by considering only LL band

2. The New concept of HVDLP is applied on LL band matrix to consider only most significant co-efficients, which leads to less number of features.

3. The LBP is used on HVDLP matrix to obtain texture features.

4. The guided filter is used with HVDLP and LBP matrix to extract final effective features. 
Signal \& Image Processing : An International Journal (SIPIJ) Vol.8, No.5, October 2017

\section{CONCLUSION}

The face images are used to identify a person efficiently. In this paper Horizontal Vertical Diagonal Local Binary Pattern based on Face Recognition is proposed. The images of different dimensions are resized to $108 \times 90$. The DWT is applied on resized image and considered only LL band of size $54 \times 45$. The new concept of HVDLP is applied on LL band to obtain feature matrix size of $36 \times 30$. The LBP is used in HVDLP matrix. The guided filter is used with HVDLP and LBP to extract final features. The ED is used to compare final features of database and test images to compute performance parameters. It is observed that the performance of the proposed methods is better compared to existing methods. In future, the classifiers, such as Neural Network and Support Vector Machines can be used for better classification of features.

\section{REFERENCES}

[1] Shengcai Liao, Xiangxin Zhu, Zhen Lei, Lun Zhang and Stan Z. Li "Learning Multi-Scale Block Local Binary Patterns for Face Recognition” Proceedings in ICB, LNCS 4642, pp. 828-837, 2007.

[2] Abdallah A. Mohamed, Darryl D'Souza, Naouel Baili and Roman V. Yampolskiy "Avatar Face Recognition using Wavelet Transform and Hierarchical Multi-scale LBP," Proceedings in 10th International Conference on Machine Learning and Applications, pp. 194-199, 2011.

[3] Timo Ojalla and MattiPietikainen "Multi resolution Grayscale and Rotation Invarient Texture Classification with Local Binary Pattern" Proceedings in IEEE Transaction on Pattern Analysis and Machine Intelligence, Vol. 24, No.7, pp.1-35, July 2002.

[4] Yu-Feng Yu, Chuan-Xian Ren, Dao-Qing Dai and Ke-Huang, "Kernel Embedding Multiorientation Local Pattern for Image Representation," Proceedings in International IEEE Transactions on Cybernetics, pp. 2168-2267, 2017.

[5] Navaveetha Bodla, Jingxiao Zheng, Honyu Xu, Jun-Cheng Chen, Carlos Castello, Rama Chellappa, "Deep Heterogeneous Feature Fusion for Template -Based face Recognition," Proceedings in IEEE Winter Conference on Applications of Computer Vision, pp. 586-595, 2017.

[6] Abdul Matin, Firoz Mahmud, Tanvir Ahmed and Md Sabbir Ejaz, "Weighted Score Level Fusion of Iris and Face to Identify an Individual," Proceedings in International IEEE Conference on Electrical, computer and Communication Engineering, pp. 1-4, 2017.

[7] Aditya Gupta, Abhijit Malage, Dhiraj More, "Feature Level Fusion of Face, Palm vein and Palmprint Modalities using Discrete Cosine Transform," Proceedings in International on Advances in Engineering Technology Research, 2014.

[8] Zhao, Chellappua, Phillip and Rosenfed, "Face Recognition : A literature Survey", Proceedings in ACM Computing Surveys, Vol. 35, No. 4, pp. 399-458, 2003.

[9] Ying Tai, Jian Yang, Yigong Zhang, Lei Luo, Jianjun Qian and Yu Chen, "Face Recognition with pose variations and Misalignment via Orthogonal Procrustes Regression," Proceedings in IEEE Transactions on Image Processing, Vol. 25, No. 6, pp. 2673-2683, 2016.

[10] Xiujuan Chai, ShiguangnShan, Xilin Chen and Wen Gao, "Locally Linear Regression for PoseInvarient Face Recognition", Proceedings in IEEE transactions on Image Processing, Vol. 16, No. 7, pp.1716-1725, 2007. 
Signal \& Image Processing : An International Journal (SIPIJ) Vol.8, No.5, October 2017

[11] Ralph Gross, Lain Matthews and Simon Baker, "Appearance-Based Face Recognition and Light Fields", Proceedings in IEEE transactions on Pattern Analysis and Machine Intelligence, Vol. 25, No. 4, pp. 449-465, 2004.

[12] Smriti Tikoo and Nitin Malik, "Detection of Face using Viola Jones and Computer Science and Mobile Computing, Vol. 5, pp. 288-295, ISSN 2320-088X, 2016.

[13] Anagha S Dhavalikar and Kulkarni "Face Detection and Facial Expression Recognition System", Proceedings in International Conference on Communication System, pp.1-7, 2014.

[14] Kavitha and Manjeet Kaur, "A Survey paper for Face Recognition Technologies", Proceedings in International Journal of Scientific and Research publication, Issue 7, ISSN 2250-3153, pp. 441-445, 2016.

[15] Samah YAHIA, Yassine Ben Salem and Mohammad Naceur ABDELKRIM, "3D Face recognition using Local Binary Pattrnand Grey Level Co-occurrence Matrix”, Proceedings in IEEE International Conference on Sciences and Techniques of Automatic Control and Computer Engineering, Sousse, T, unisia, pp. 328-338, 2016.

[16] ORL Database, http://www.camrol.co.uk

[17] Indian Face Database, http://viswww.cs.umass.edu/ vidit/Indian Face Database

[18] Jagadeesh H S, Suresh Babu K and Raja KB, "DBC based Face Recognition using DWT," Proceedings in International Journal of Signal and Image Processing, Vol 3, pp.115-129, 2012.

[19] T. Ahonen,, A. Hadid and Pietikainen, "Face Description with Local Binary Patterns," Application to face recognition, TPAMI, (28)12, 2017-2041, 2006.

[20] Keiming He, Jian Sun, Xiaoog Tang, "Guided Image Filtering," Proceedings in International Journal of IEEE Transactions on Pattern Analysis and Machine Intelligence, Vol. 35, pp. 1-13, 2013.

[21] D Murugan, S Arumugam, K Rajalakshmi and Manish T, "Performance Evaluation of Face Recognition using Gabor Filter, Log Gabor filter and Discrete Wavelet Transform," Proceedings in International Journal of computer science and Information Technology, Vol.2(1), pp. 125-133, 2010.

[22] Pallavi D.Wadkar and Megha Wankhade, Face Recognition using Discrete Wavelet Transforms, Proceedings in International Journal of Advanced Engineering Technology, vol.3, pp. 239-242, 2012.

[23] Sujatha B M, K Suresh Babu, K B Raja, Venugopal K R, Hybrid Domain based Face Recognition using DWT, FFT and Compressed CLBP, Proceedings in International Journal of Image processing (IJIP), Vol.9, pp. 283-303, 2015.

\section{AUTHORS}

Mrs Chandrakala $\mathbf{V}$ received B.E (Electronics and Communication) Bangalore University, M. Tech., (Digital Communication) Visvesvaraya Technological University Belgaum. Currently pursuing Ph.D degree from Bangalore University, Bangalore, working as Associate Professor, department of Telecommunication engineering at Dr. Ambedkar Institute of Technology, Bangalore. Life member of Indian Society for Technical Education(ISTE) New Delhi and Institute of engineers(IE).

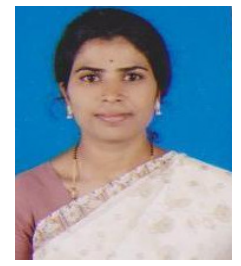


Signal \& Image Processing : An International Journal (SIPIJ) Vol.8, No.5, October 2017

The research areas include image Processing, Patten recognition, Biometrics and Networking and having 15 years of teaching experience in engineering college.

Venukumar $\mathbf{B} \mathbf{V}$ is an active learner and researcher in the field of electronics. $\mathrm{He}$ obtained Bachelor of Engineering (B.E) degreein Electronics and Telecommunication from B.N.M Institute of Technology, Bengaluru and currently pursuing Master of Engineering (M.E) degree in Electronics and Communication from University Visvesvaraya College of Engineering (U.V.C.E), Bangalore University, Bengaluru. He has done over 5 projects on Biometrics. His research work has been accepted and presented on an esteemed Twelfth International Conference ELSEVIER-2016. He has guided over 20 UG students on Image processing and MATLAB coding, and enjoys sharing his knowledge. His research interest includes Image Processing, Digital Signal Processing, Biometrics and Embedded Systems.

K Suresh Babu is Professor, Dept. of Electronics and Communication Engineering, University Visvesvaraya College of Engineering, Bangalore University, Bangalore. He obtained his $\mathrm{BE}$ and $\mathrm{ME}$ in Electronics and Communication Engineering from University Visvesvaraya College of Engineering, Bangalore. He was awarded Ph.D. in Computer Science and Engineering from Bangalore University. He has over 25 research publications in refereed International Journals and Conference Proceedings. His research interests include Image Processing, Biometrics, Signal Processing,

K B Raja is Professor, Chairman and HoD, Dept. of Electronics and Communication Engineering, University Visvesvaraya College of Engineering, Bangalore University, Bangalore. He obtained his BE and ME in Electronics and Communication Engineering from University Visvesvaraya College of Engineering, Bangalore. He was awarded $\mathrm{Ph} . \mathrm{D}$. in Computer Science and Engineering from Bangalore University. He has over 180 research publications in refereed International Journals and Conference Proceedings. His research interests include Image Processing, Biometrics, VLSI Signal Processing, computer networks.

K R Venugopal is currently the chairman, University Visvesvaraya College of Engineering, Bangalore University, Bangalore. He obtained his Bachelor of Engineering from University Visvesvaraya College of Engineering. He received his Masters degree in Computer Science and Automation from Indian Institute of Science, Bangalore. He was awarded Ph.D. in Economics from Bangalore University and Ph.D. in Computer Science from Indian Institute of Technology, Madras. He has over 600 research publications in refereed International Journals and Conference Proceedings. His research interests include computer networks, parallel and distributed systems, digital signal
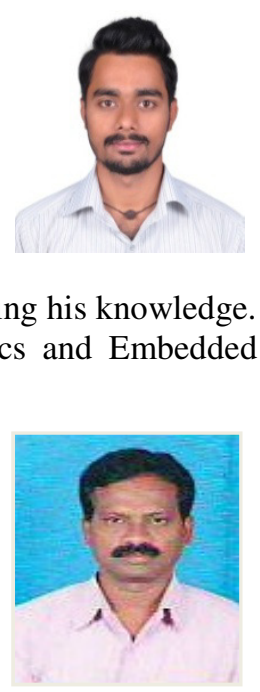
processing and data mining. 